\title{
Recent developments of the projected shell model based on many-body tech- niques
}

\author{
Yang Sun ${ }^{1, a}$, Long-Jun Wang ${ }^{1}$, Fang-Qi Chen ${ }^{1,2}$, Takahiro Mizusaki ${ }^{3}$, Makito $\mathrm{Oi}^{3}$, and Peter Ring ${ }^{4}$ \\ ${ }^{1}$ Department of Physics and Astronomy, Shanghai Jiao Tong University, Shanghai 200240, People's Republic of China \\ ${ }^{2}$ China Institute of Atomic Energy, Beijing 102413, People's Republic of China \\ ${ }^{3}$ Institute of Natural Sciences, Senshu University, 3-8-1 Kanda-Jinbocho, Chiyoda-ku, Tokyo 101-8425, Japan \\ ${ }^{4}$ Physik-Department der Technischen Universität München, D-85748 Garching, Germany
}

\begin{abstract}
Recent developments of the projected shell model (PSM) are summarized. Firstly, by using the Pfaffian algorithm, the multi-quasiparticle configuration space is expanded to include 6-quasiparticle states. The yrast band of ${ }^{166} \mathrm{Hf}$ at very high spins is studied as an example, where the observed third back-bending in the moment of inertia is well reproduced and explained. Secondly, an angular-momentum projected generate coordinate method is developed based on PSM. The evolution of the low-lying states, including the second $0^{+}$state, of the soft Gd, Dy, and Er isotopes to the well-deformed ones is calculated, and compared with experimental data.
\end{abstract}

\section{Introduction}

Nuclei are among the few quantum systems that can be discussed in terms of shape [1]. It is well-known that most nuclei in the nuclear chart are deformed. The most popular type of deformation is axial-symmetric quadrupole deformation. Calculations of total energies for such nuclei usually show a pronounced minimum at a certain deformation. Figure 1 illustrates a representative example from the ${ }^{254}$ No calculation, a well-deformed heavy nucleus in the transfermium region. It is seen that deep minima are robustly developed at the deformation $\varepsilon_{2} \approx+0.25$, no matter if the calculation is performed with (solid curves) or without (dotted curve) angular momentum projection. Other local minima are well separated, lying by several $\mathrm{MeV}$ higher above the ground state. Therefore, this nucleus can be well regarded as a prolately-deformed rotor in the classical picture. In fact, a regular rotational band, with the band energies following the $\sim I(I+1)$ dependence expected from those of a classical rotor, has been observed experimentally [2].

Physics for such regularly-deformed nuclei can be described by models that choose the known deformation to construct a deformed basis. In their ground state, nuclei tend to couple their nucleons pairwise due to the pairing correlation. Thus, a deformed-quasiparticle (qp) basis obtained from the Nilsson+BCS calculation can be a good starting point to model such nuclear systems. Angularmomentum and particle-number projection can be incorporated, respectively, to recover the violated rotational and gauge symmetries in the Nilsson and BCS calculations.

\footnotetext{
ae-mail: sunyang@sjtu.edu.cn
}

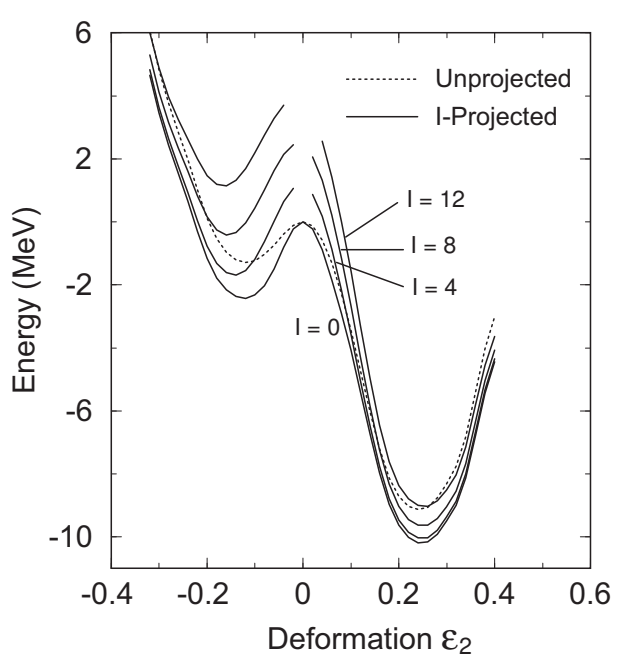

Figure 1. Calculated angular-momentum-projected energy surfaces for ${ }^{254} \mathrm{No}$ as functions of the quadrupole deformation parameter. The unprojected calculation is given by the dotted curve.

Based on the deformed qp-vacuum, other configurations of qp excitations are included in the basis, and mixing of all these configurations is done by the shell model diagonalization. This is the basic philosophy that the Projected Shell Model (PSM) follows [3].

Nuclear rotation tends to break the nucleon pairs due to the Coriolis antipairing effect [4]. The nucleon pairs in the orbitals with the highest angular momentum $j$, as for instance the neutron $i_{13 / 2}$ shell in the rare earth region, feel a stronger Coriolis force, and therefore, break first [5]. They contribute to the formation of a 2-qp state as the main con- 


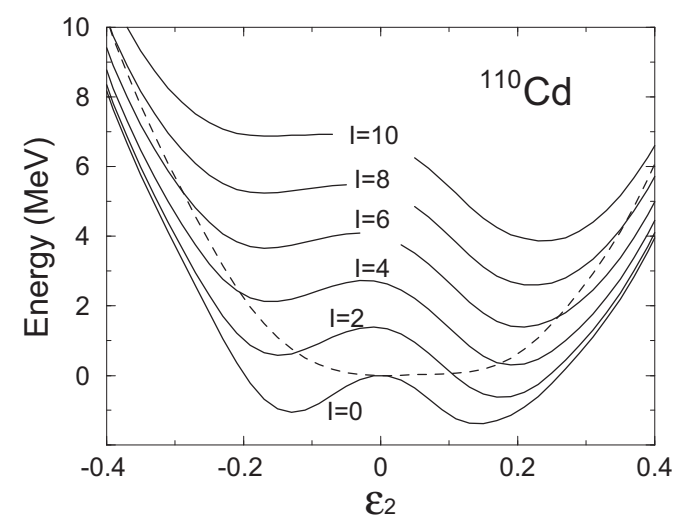

Figure 2. Calculated angular-momentum-projected energy surfaces for ${ }^{110} \mathrm{Cd}$ as functions of the quadrupole deformation parameter. The unprojected calculation is given by the dashed curve.

figuration of the yrast state. As a nucleus rotates faster and faster, subsequent pair-breakings can occur for the pairs from the next highest $j$ orbitals. In the rare earth region, proton pairs in the $h_{11 / 2}$ shell are expected to break next. Further pair breakings at higher angular momenta are possible, and there have been early [6] and recent evidences $[7,8]$ of breaking of three nucleon pairs, which form 6-qp states as the main configuration of the yrast sequence.

The difficulty to describe the above phenomena in a shell-model framework lies in the procedure of computing the overlap matrix elements [4] between arbitrary multiqp states. Since the involved overlap matrix elements of multi-qp states are usually calculated with the generalized Wick's theorem [3], one may encounter a problem of combinatorial complexity when more than 4-qp states are included in the basis configurations. Therefore, to push the calculation further involving higher order of qp states, a breakthrough in computational many-body techniques is needed. In nuclear structure physics, the Pfaffian concept has been introduced [9] as a key mathematical tool for solving the long-standing problem in the phase determination of the Onishi formula [10]. Moreover, it has been shown that the Pfaffian algorithm is very efficient also for calculating overlap matrix elements [11-16]. In particular, by means of Fermion coherent states and the Grassmann integral, one can derive an alternative approach to calculate the rotated matrix element for general qp states [17], which serves as a theoretical framework to extend the PSM model space [18].

However, there are many other nuclei either having multiple shapes coexisting near the ground state or being very soft without any simply-defined shapes [19]. Figure 2 illustrates an example from the ${ }^{110} \mathrm{Cd}$ calculation, a soft nucleus in the transitional region. It is seen that for this nucleus, the angular-momentum-projected and unprojected calculations show qualitatively different features. While the unprojected calculation (dashed curve in Fig. 2) suggests a spherical shape, the ground state obtained by the projected calculation shows competing prolate-oblate minima separated by only a small energy barrier. More- over, as the nucleus rotates, the minima change towards larger absolute values; namely, the nucleus becomes more and more deformed [20]. Obviously for this kind of nuclei, one can not build a shell model basis simply with one deformation. The correct way to describe such systems is to superimpose in the wavefunction all possible shapes described by different deformation parameters, which is a concept generally known in the literature as the Generater Coordinate Method (GCM) [4].

The present paper summaries the recent development of PSM based on the Pfaffian algorithm and the projected Generater Coordinate Method.

\section{Application of the Pfaffian algorithm}

\subsection{The formalism}

The PSM employs the Nilsson model [21] to generate a deformed single-particle basis. Pairing correlations are considered by a BCS calculation. The Nilsson-BCS calculation defines a deformed qp basis from which the PSM model space is constructed. The multi-qp configurations up to 6-qp states for even-even nuclei are given as [18]

$$
\begin{aligned}
& \left\{|\Phi\rangle, a_{v_{i}}^{\dagger} a_{v_{j}}^{\dagger}|\Phi\rangle, a_{\pi_{i}}^{\dagger} a_{\pi_{j}}^{\dagger}|\Phi\rangle, a_{v_{i}}^{\dagger} a_{v_{j}}^{\dagger} a_{\pi_{k}}^{\dagger} a_{\pi_{l}}^{\dagger}|\Phi\rangle,\right. \\
& \quad a_{v_{i}}^{\dagger} a_{v_{j}}^{\dagger} a_{v_{k}}^{\dagger} a_{v_{l}}^{\dagger}|\Phi\rangle, a_{\pi_{i}}^{\dagger} a_{\pi_{j}}^{\dagger} a_{\pi_{k}}^{\dagger} a_{\pi_{l}}^{\dagger}|\Phi\rangle, \\
& a_{v_{i}}^{\dagger} a_{v_{j}}^{\dagger} a_{v_{k}}^{\dagger} a_{v_{l}}^{\dagger} a_{v_{m}}^{\dagger} a_{v_{n}}^{\dagger}|\Phi\rangle, a_{\pi_{i}}^{\dagger} a_{\pi_{j}}^{\dagger} a_{\pi_{k}}^{\dagger} a_{\pi_{l}}^{\dagger} a_{\pi_{m}}^{\dagger} a_{\pi_{n}}^{\dagger}|\Phi\rangle, \\
& \left.a_{\pi_{i}}^{\dagger} a_{\pi_{j}}^{\dagger} a_{v_{k}}^{\dagger} a_{v_{l}}^{\dagger} a_{v_{m}}^{\dagger} a_{v_{n}}^{\dagger}|\Phi\rangle, a_{v_{i}}^{\dagger} a_{v_{j}}^{\dagger} a_{\pi_{k}}^{\dagger} a_{\pi_{l}}^{\dagger} a_{\pi_{m}}^{\dagger} a_{\pi_{n}}^{\dagger}|\Phi\rangle\right\} .
\end{aligned}
$$

In the above expression, $a_{v}^{\dagger}, a_{\pi}^{\dagger}\left(a_{v}, a_{\pi}\right)$ denote neutron and proton qp creation (annihilation) operators associated with the qp vacuum $|\Phi\rangle$.

The PSM wave function is a linear combination of projected states

$$
\left|\Psi_{I M}^{\sigma}\right\rangle=\sum_{K \kappa} f_{I K_{K}}^{\sigma} \hat{P}_{M K}^{I}\left|\Phi_{\kappa}\right\rangle
$$

where $\left|\Phi_{K}\right\rangle$ are the qp-states in (1). $\hat{P}_{M K}^{I}$ is the angular momentum projection operator [4]

$$
\hat{P}_{M K}^{I}=\frac{2 I+1}{8 \pi^{2}} \int d \Omega D_{M K}^{I}(\Omega) \hat{R}(\Omega),
$$

with $D_{M K}^{I}$ being the $D$-function, $\hat{R}$ the rotation operator, and $\Omega$ the Euler angles. The energies and wave functions are obtained by solving the eigenvalue equation:

$$
\sum_{K^{\prime} \kappa^{\prime}}\left(H_{K \kappa, K^{\prime} \kappa^{\prime}}^{I}-E_{I}^{\sigma} N_{K \kappa, K^{\prime} \kappa^{\prime}}^{I}\right) f_{I K_{\kappa^{\prime}}^{\prime}}^{\sigma}=0,
$$

where $H_{K \kappa, K^{\prime} \kappa^{\prime}}^{I}$ and $N_{K \kappa, K^{\prime} \kappa^{\prime}}^{I}$ are the projected matrix elements of the Hamiltonian and the norm respectively

$$
H_{K \kappa, K^{\prime} K^{\prime}}^{I}=\left\langle\Phi_{\kappa}\left|\hat{H} \hat{P}_{K K^{\prime}}^{I}\right| \Phi_{\kappa^{\prime}}\right\rangle, \quad N_{K \kappa, K^{\prime} K^{\prime}}^{I}=\left\langle\Phi_{\kappa}\left|\hat{P}_{K K^{\prime}}^{I}\right| \Phi_{K^{\prime}}\right\rangle .
$$

The central task in numerical calculations is to evaluate rotated matrix elements of the Hamiltonian and the norm

$$
\mathcal{H}_{\kappa K^{\prime}}=\left\langle\Phi_{\kappa}|\hat{H}[\Omega]| \Phi_{\kappa^{\prime}}\right\rangle, \quad \mathcal{N}_{\kappa K^{\prime}}=\left\langle\Phi_{\kappa}|[\Omega]| \Phi_{\kappa^{\prime}}\right\rangle,
$$


with the operator $[\Omega]=\hat{R}(\Omega) /\langle\Phi|\hat{R}(\Omega)| \Phi\rangle$ [3]. Since $\mathcal{H}_{\kappa K^{\prime}}$ can be decomposed into terms expressed by the "linked" contraction and $\mathcal{N}_{\kappa K^{\prime}}$ [3], the main task then concentrates on treating efficiently $\mathcal{N}_{\kappa K^{\prime}}$. For the sake of convenience, we rewrite it as the following explicit form

$$
\mathcal{N}_{\kappa K^{\prime}}=\left\langle\Phi\left|a_{1} \cdots a_{n}[\Omega] a_{1^{\prime}}^{\dagger} \cdots a_{n^{\prime}}^{\dagger}\right| \Phi\right\rangle,
$$

which is usually evaluated [3] by the generalized Wick's theorem that decomposes Eq. (7) into a combination of three types of contractions, denoted as $A, B$, and $C$, with their matrix expressions [22]

$$
\begin{aligned}
& A_{v v^{\prime}}(\Omega) \equiv\left\langle\Phi\left|[\Omega] a_{v^{\prime}}^{\dagger} a_{v^{\prime}}^{\dagger}\right| \Phi\right\rangle, \\
& B_{v v^{\prime}}(\Omega) \equiv\left\langle\Phi\left|a_{v} a_{v^{\prime}}[\Omega]\right| \Phi\right\rangle, \\
& C_{v v^{\prime}}(\Omega) \equiv\left\langle\Phi\left|a_{v}[\Omega] a_{v^{\prime}}^{\dagger}\right| \Phi\right\rangle .
\end{aligned}
$$

It was pointed out [17] that in applying the generalized Wick's theorem, a matrix element of Eq. (7) involving $n$ and $n^{\prime}$ qps, respectively in the left- and right-side of [ $\left.\Omega\right]$, contains $\left(n+n^{\prime}-1\right)$ !! terms. In practice, the number of terms becomes so large that it is nearly impossible to write down expressions explicitly for more than 4-qp states.

By using the Fermion coherent state and the Grassmann integral, a general expression for the matrix elements (7) in terms of the Pfaffian can be derived [17]

$$
\left\langle\Phi\left|a_{1} \cdots a_{n}[\Omega] a_{1^{\prime}}^{\dagger} \cdots a_{n^{\prime}}^{\dagger}\right| \Phi\right\rangle=\operatorname{Pf}(X)=\operatorname{Pf}\left(\begin{array}{cc}
B & C \\
-C^{T} & A
\end{array}\right),
$$

where $X$ is a skew-symmetric matrix with dimension $(n+$ $\left.n^{\prime}\right) \times\left(n+n^{\prime}\right)$. The indices of rows and columns for $B$ run from 1 to $n(1, \ldots, n)$ and the ones for $A$ run from $1^{\prime}$ to $n^{\prime}\left(1^{\prime}, \ldots, n^{\prime}\right)$. For the matrix $C$ in Eq. (9), the indices of rows run from 1 to $n$ and those of columns run from $1^{\prime}$ to $n^{\prime}$. The Pfaffian is defined as

$$
\operatorname{Pf}(\mathcal{A}) \equiv \frac{1}{2^{n} n !} \sum_{\sigma \in S_{2 n}} \operatorname{sgn}(\sigma) \prod_{i=1}^{n} a_{\sigma(2 i-1) \sigma(2 i)} .
$$

for a skew-symmetric matrix $\mathcal{A}$ with dimension $2 n \times 2 n$, of which matrix elements are $a_{i j}$. The symbol $\sigma$ is a permutation of $\{1,2,3, \ldots, 2 n\}, \operatorname{sgn}(\sigma)$ is its sign, and $S_{2 n}$ represents a symmetry group. This makes it possible and efficient to work with the expanded PSM configuration in (1), since calculations of the corresponding Pfaffian are not time-consuming [23].

\subsection{The ${ }^{166} \mathrm{Hf}$ example}

The PSM employs the Hamiltonian with separable forces:

$$
\hat{H}=\hat{H}_{0}-\frac{1}{2} \chi_{Q Q} \sum_{\mu} \hat{Q}_{2 \mu}^{\dagger} \hat{Q}_{2 \mu}-G_{M} \hat{P}^{\dagger} \hat{P}-G_{Q} \sum_{\mu} \hat{P}_{2 \mu}^{\dagger} \hat{P}_{2 \mu}
$$

where $\hat{H}_{0}$ is the spherical single-particle term including the spin-orbit force [24], and the rest is the quadrupole+pairing type of interactions, which contains

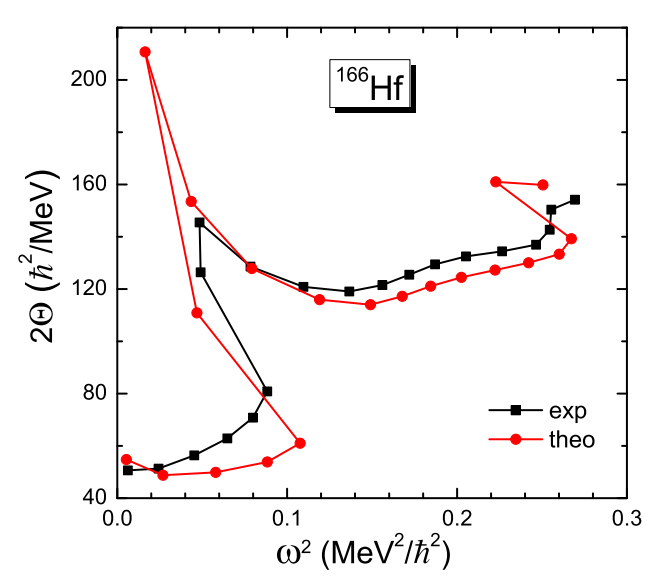

Figure 3. (Color online) Back-bending plot for ${ }^{166} \mathrm{Hf}$. The calculated results are compared with the data taken from Ref. [7]. This figure is taken from Fig. 1 of Ref. [18].

three parts. The strength of the quadrupole-quadrupole term $\chi_{Q Q}$ is related to deformation of the basis [3]. The monopole-pairing strength is taken to be the form $G_{M}=$ $\left[G_{1} \mp G_{2}(N-Z) / A\right] / A$, where "+" ("-") is for protons (neutrons), with $G_{1}=20.12$ and $G_{2}=13.13$ being the coupling constants [3], in correspondence to the full configuration space of three major harmonic-oscillator shells, $N=4,5,6(N=3,4,5)$ for neutrons (protons). The quadrupole-pairing strength $G_{Q}$ is taken, as usual, to be $16 \%$ of $G_{M}$ for all the nuclei considered in this study.

The anomalies in the observed moment of inertia (MOI) of a rotating nucleus are usually displayed in an exaggerated manner with a back-bending plot, in which twice the MOI, $2 \Theta$, is plotted as a function of the square of the rotational frequency $\omega^{2}$. Figure 3 shows the backbending plot for ${ }^{166} \mathrm{Hf}$, where the theoretical results are compared with the experimental data. In the calculation, the deformation parameters are fixed as $\varepsilon_{2}=0.208$ and $\varepsilon_{4}=0.013$ which are taken from Ref. [25]. Anomalies in MOI can be clearly seen as $\omega$ increases, roughly at $\omega^{2} \approx 0.10,0.15$ and 0.25 , corresponding to $\operatorname{spin} I \approx 12,24$ and 34 , respectively. The first anomaly exhibits the largest effect, causing a sharp increase in $2 \Theta$. This is known as the first back-bending, corresponding to breaking and alignment of a neutron $i_{13 / 2}$ pair. The second anomaly in Fig. 3 corresponds to the small increase in $2 \Theta$ at $\omega^{2} \approx 0.15$, which is nicely reproduced by the calculation. At this rotational frequency, an additional $h_{11 / 2}$ proton pair is broken and their spins are aligned along the axis of rotation. The third anomaly belongs to the few known cases that have ever been observed: $2 \Theta$ jumps suddenly again at $\omega^{2} \approx 0.25$. The observation is correctly described by the present calculation, and is understood as the additional breaking of a second neutron $i_{13 / 2}$ pair. Therefore above this point the yrast line consists of a 6-qp configuration with two qp-pairs in the neutron $i_{13 / 2}$ shell and one qp-pair in the proton $h_{11 / 2}$ shell.

The calculated results can be analyzed by the band diagram [3], where energies of the theoretical bands are plotted as functions of spin. The energy of band $\kappa$ is defined 


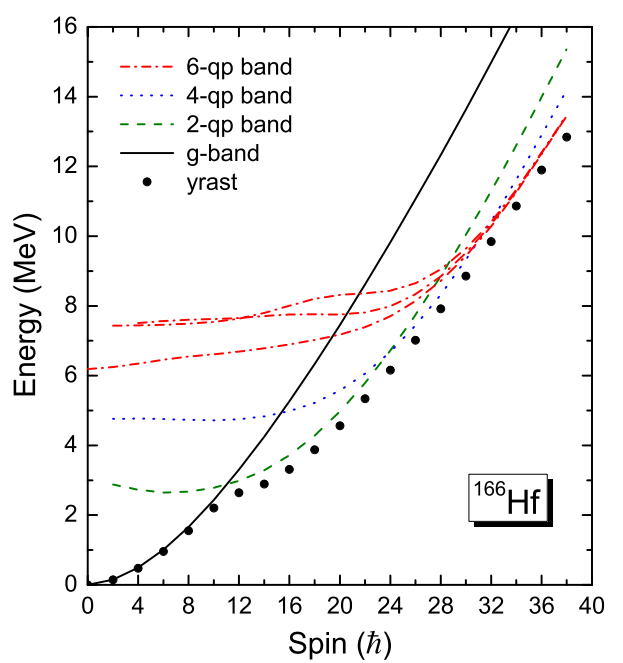

Figure 4. (Color online) Band diagram for ${ }^{166} \mathrm{Hf}$. Note that only even-spin states are plotted in order to avoid zigzag in these curves. This figure is taken from Fig. 2 of Ref. [18].

as

$$
E_{K}(I)=\frac{\left\langle\Phi_{\kappa}\left|\hat{H} \hat{P}_{K K}^{I}\right| \Phi_{K}\right\rangle}{\left\langle\Phi_{K}\left|\hat{P}_{K K}^{I}\right| \Phi_{\kappa}\right\rangle}
$$

which is the projected energy of a multi-qp configuration in (1). Figure 4 displays the band diagram for ${ }^{166} \mathrm{Hf}$, where the 0-qp ground (g-) band, one 2-qp band, one 4-qp band, and three 6 -qp bands are selected from about 200 projected configurations because of their important roles played in the yrast band (marked by dots). It is seen that the first back-bending at $I \approx 12$ in Fig. 3 corresponds to the crossing between $\mathrm{g}$-band and the 2 -qp $(s-)$ band. The configuration of the $s$-band is found to be $v 3 / 2^{+}[651] \otimes v 5 / 2^{+}[642]$ with $K=1$. The $s$-band remains to be the yrast band until it is crossed by a 4-qp band at $I \approx 24$. This 4qp band is based on an addition of an $h_{11 / 2}$ proton pair, corresponding to the configuration $v 3 / 2^{+}[651] 5 / 2^{+}[642] \otimes$ $\pi 7 / 2^{-}[523] 9 / 2^{-}[514]$ with $K=2$. The third anomaly in MOI corresponds to the crossing of the 4-qp band with three 6 -qp bands at $I \approx 34$. Two of the 6-qp bands whose energies are almost the same at low spins have the same configuration $v 1 / 2^{+}[660] 3 / 2^{+}[651] 5 / 2^{+}[642] 7 / 2^{+}[633] \otimes$ $\pi 7 / 2^{-}[523] 9 / 2^{-}[514]$ but with different $K$ values $K=$ -1 and -3 . The configuration of the third 6qp band is $v 1 / 2^{+}[660] 3 / 2^{+}[651] 5 / 2^{+}[642] 5 / 2^{+}[642] \otimes$ $\pi 7 / 2^{-}[523] 9 / 2^{-}[514]$ with $K=0$. As the level density increases with spin, the wave function of the yrast band beyond $I \approx 34$ is found to have a large admixture of these 6-qp states.

\section{Generater Coordinate Method}

While the above discussed method is powerful for nuclei with a well-defined stable shape, it can not be applied to transitional nuclei which either have multiple shapes coexisting near the ground state or are so soft that one can not even talk above shapes. Quite recently, we have improved
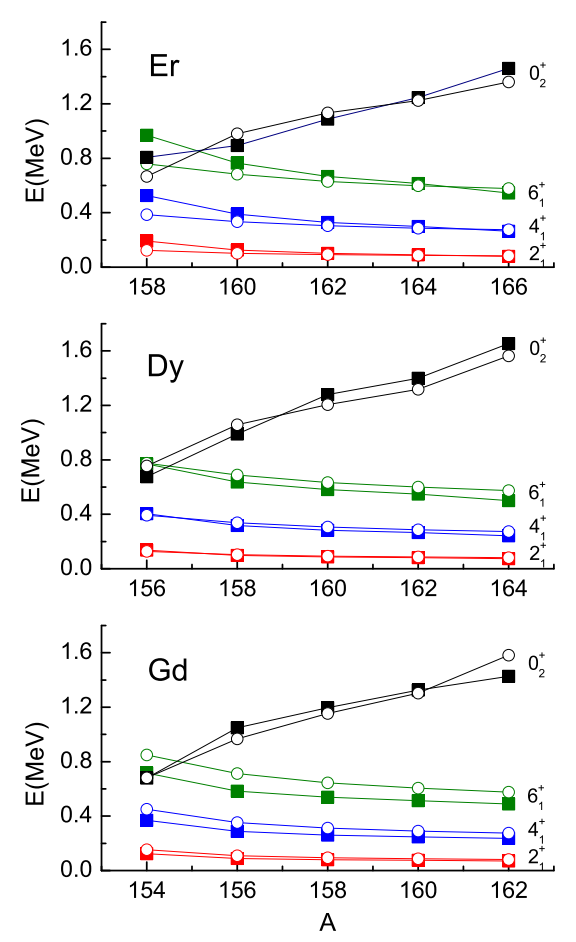

Figure 5. (Color online) Comparison of the calculated $0_{2}^{+}$state with experimental data for $\mathrm{Gd}$, Dy, and Er isotopes. The energy levels $2_{1}^{+}, 4_{1}^{+}$, and $6_{1}^{+}$of the ground-state rotational band are also shown. Calculated results (open circles) are compared with data (filled squares). This figure is taken from Fig. 1 of Ref. [26].

the PSM wavefunction by using the Generater Coordinate Method (GCM) [26].

\subsection{GCM Theory}

To improve the wavefunction (2) which corresponds to a fixed quadrupole deformation $\varepsilon_{2}$, a better wave function can be constructed by taking $\varepsilon_{2}$ as the generate coordinate

$$
\left|\Psi^{I, N}\right\rangle=\int d \varepsilon_{2} f^{I, N}\left(\varepsilon_{2}\right) \hat{P}^{I} \hat{P}^{N}\left|\Phi\left(\varepsilon_{2}\right)\right\rangle,
$$

where $\hat{P}^{N}$ is the particle-number projection operators. The weight $f^{I, N}\left(\varepsilon_{2}\right)$ is determined by the diagolnalization.

This method has been used for the investigations of $0^{+}$ excitations also in the context of non-relativistic [27, 28] and relativistic [29] Density Functional Theory (DFT). These are extremely time-consuming calculations, and therefore in many cases additional approximations are used to derive the parameters of a Bohr-Hamiltonian, as for instance the Gaussion Overlap Approximation (GOA) or Adiabatic Time-Dependent Hartree Fock (ATDHF) theory $[29,30]$. Because of the small effective mass in many density functional theories [31] the corresponding single particle spectra deviate considerably from experimental values. The advantage of our approach is its simplicity together with the realistic single particle spectra of the Nilsson model [21] used here. 


\subsection{GCM examples}

In Fig. 5, we show the results of a systematic calculation for the first excited $0_{2}^{+}$state in $\mathrm{Gd}$, Dy, and Er isotopes, and compare them with experimental data. The energy levels $2_{1}^{+}, 4_{1}^{+}$, and $6_{1}^{+}$of the ground state rotational band are also shown. We find that with a single set of parameters in the Hamiltonian, the characteristic behavior of the shell evolution is well reproduced. This certainly benefits from the improved GCM wave function of Eq. (13). It is seen that starting from the neutron number $N=90$ on the 1.h.s. of Fig. 5 and going to heavier isotopes the ground state bands becomes more and more compressed, eventually following the rotational rule of $E \sim I(I+1)$ at $N=98$. On the other hand, the $0_{2}^{+}$state is found low for the isotopes with neutron number 90, which are thought to be soft nuclei with low-energy vibrational states. Without any adjustable parameter, the increase of the energy of the $0_{2}^{+}$state with increasing neutron number is correctly reproduced for all three isotopic chains, thus clearly distinguishing the spectral differences of softness in lighter isotopes and stiffness in heavier isotopes, with regard to deformation. The case with the largest discrepancy between calculation and data is ${ }^{158} \mathrm{Er}$, which is known to be relatively soft in triaxiality. We may conclude that the improved GCM wave function of Eq. (13) is able to describe correctly both soft and stiff nuclei, and the transition between them.

\section{Summary}

This paper summarizes the recent developments of PSM by using the many-body techniques, which allow to describe rotationally induced structural changes at very high spins and shape evolutions between the soft to welldeformed nuclei. On one hand, the configuration space of PSM has been expanded considerably by using the Pfaffian algorithm. On the other hand, the angular-momentumprojected GCM has been developed based on the PSM framework, which largely enriched the PSM wavefunctions. A combination of these two developments, namely the construction of multi-qp configurations on top of the GCM states, is under our consideration.

\section{Acknowledgements}

Research at SJTU was supported by the National Natural Science Foundation of China (No. 11135005) and by the 973 Program of China (No. 2013CB834401).

\section{References}

[1] A. Bohr and B. R. Mottelson, Nuclear Structure, Vol. 2 (Benjamin, New York, 1975)
[2] R.-D. Herzberg and P. T. Greenlees, Prog. Part. Nucl. Phys. 61, 674 (2008)

[3] K. Hara and Y. Sun, Int. J. Mod. Phys. E 4, 637 (1995)

[4] P. Ring and P. Schuck, The nuclear many-body problem (Springer Verlag, Berlin, 2004)

[5] F. S. Stephens and R. S. Simon, Nucl. Phys. A 183, 257 (1972)

[6] J. Burde et al., Phys. Rev. Lett. 48, 530 (1982)

[7] D. R. Jensen et al., Eur. Phys. J. A 8, 165 (2000)

[8] R. B. Yadav et al., Phys. Rev. C 80, 064306 (2009)

[9] L. M. Robledo, Phys. Rev. C 79, 021302(R) (2009)

[10] N. Onishi and S. Yoshida, Nucl. Phys. 80, 367 (1966)

[11] L. M. Robledo, Phys. Rev. C 84, 014307 (2011)

[12] G. F. Bertsch and L. M. Robledo, Phys. Rev. Lett. 108, 042505 (2012)

[13] B. Avez and M. Bender, Phys. Rev. C 85, 034325 (2012)

[14] M. Oi and T. Mizusaki, Phys. Lett. B 707, 305 (2012)

[15] T. Mizusaki and M. Oi, Phys. Lett. B 715, 219 (2012)

[16] Q.-L. Hu, Z.-C. Gao, and Y. S. Chen, Phys. Lett. B 734, 162 (2014)

[17] T. Mizusaki, M. Oi, F. Q. Chen, and Y. Sun, Phys. Lett. B 725, 175 (2013)

[18] L.-J. Wang, F.-Q. Chen, T. Mizusaki, M. Oi, and Y. Sun, Phys. Rev. C 90, 011303(R) (2014)

[19] K. Heyde and J. L. Wood, Rev. Mod. Phys. 83, 1467 (2011)

[20] P. H. Regan et al., Phys. Rev. Lett. 90, 152502 (2003)

[21] S. G. Nilsson et al., Nucl. Phys. A 131, 1 (1969).

[22] K. Hara and S. Iwasaki, Nucl. Phys. A 332, 61 (1979).

[23] C. González-Ballestero, L. M. Robledo, and G. F. Bertsch, Comput. Phys. Commun. 182, 2213 (2011)

[24] T. Bengtsson and I. Ragnarsson, Nucl. Phys. A 436, 14 (1985)

[25] P. Moller, J. R. Nix, W. D. Myers, and W. J. Swiatecki, At. Data Nucl. Data Tables 59, 185 (1995)

[26] F.-Q. Chen, Y. Sun, and P. Ring, Phys. Rev. C 88, 014315 (2013)

[27] T. R. Rodríguez and J. L. Egido, Phys. Lett. B 705, 255 (2011)

[28] J. M. Yao, M. Bender, and P.-H. Heenen, Phys. Rev. C 87, 034322 (2013)

[29] Y. Fu et al., Phys. Rev. C 87, 054305 (2013)

[30] J.-P. Delaroche et al., Phys. Rev. C 81, 014303 (2010)

[31] E. Litvinova and P. Ring, Phys. Rev. C 73, 044328 (2006) 
